\title{
EXTENSION PROBLEM OF SEVERAL CONTINUITIES IN COMPUTER TOPOLOGY
}

\author{
SANG-EON HAN
}

\begin{abstract}
The goal of this paper is to study extension problems of several continuities in computer topology. To be specific, for a set $X \subset \mathbf{Z}^{n}$ take a subspace $\left(X, T_{X}^{n}\right)$ induced from the Khalimsky $n$ D space $\left(\mathbf{Z}^{n}, T^{n}\right)$. Considering $\left(X, T_{X}^{n}\right)$ with one of the $k$-adjacency relations of $\mathbf{Z}^{n}$, we call it a computer topological space (or a space if not confused) denoted by $X_{n, k}$. In addition, we introduce several kinds of $k$-retracts of $X_{n, k}$, investigate their properties related to several continuities and homeomorphisms in computer topology and study extension problems of these continuities in relation with these $k$-retracts.
\end{abstract}

\section{Introduction}

Let $\mathbf{N}, \mathbf{Z}, \mathbf{R}$ and $\mathbf{Z}^{n}$ be the sets of natural numbers, integers, real numbers and points in the Euclidean $n \mathrm{D}$ space with integer coordinates, respectively. The extension problem well known is also very important in applied topology including digital topology [20], Scott topology [5] and computer topology [7, $10,15]$ and further, we can expand the problem with various continuities in [10]. More precisely, for sets $X \subset \mathbf{Z}^{n_{0}}$ and $Y \subset \mathbf{Z}^{n_{1}}$ we remind that for a map $f: X \rightarrow Y$ a map $F: X^{\prime} \rightarrow Y$ is an extension of $f$ if the restriction map $F$ on $X \subset X^{\prime}$, briefly $\left.F\right|_{X}$, is equal to $f$.

The paper begins with a brief review of some digital topological results of an extension problem. Motivated by the extension problem in [2], the paper [3] established a digital $k$-retract for studying an extension problem of digital continuity. In digital topology we have usually studied a set $X \subset \mathbf{Z}^{n}$ with one of the $k$-adjacency relations of $\mathbf{Z}^{n}$ (briefly, a digital space) denoted by $(X, k)$.

Received December 30, 2008.

2000 Mathematics Subject Classification. 54C20, 54C05, 54D05, 54F05, 68U05.

Key words and phrases. computer topology, digital topology, extension problem, Khalimsky topology, computer topological continuity, computer topological homeomorphism, $k$ retract.

This research was supported by Basic Science Research Program through the National Research Foundation of Korea(NRF) funded by the Ministry of Education, Science and Technology(20090067572).

This paper was supported by the selection of research-oriented professor of Chonbuk National University in 2010 . 
For a map $f:\left(X, k_{0}\right) \rightarrow\left(Y, k_{1}\right)$, since a preservation of the $k_{0}$-connectivity of $\left(X, k_{0}\right)$ into the $k_{1}$-one of $\left(Y, k_{1}\right)$ can strongly contribute to the study of a digital space, digital $\left(k_{0}, k_{1}\right)$-continuity has been used in studying digital spaces $[3,7,9,11,20]$.

As another digital topological approach, we have often considered a set $X \subset \mathbf{Z}^{n}$ to be a subspace induced from the Khalimsky $n \mathrm{D}$ space $\left(\mathbf{Z}^{n}, T^{n}\right)$ denoted by $\left(X, T_{X}^{n}\right)$. The paper [19] studied an extension problem of a continuous function from a subspace of the Khalimsky $n \mathrm{D}$ space to the Khalimsky line. When studying a Khalimsky topological space, a preservation of a digital connectivity is also important. However, if a Khalimsky topological space $\left(X, T_{X}^{n}\right)$ is not connected from the view point of Khalimsky topology, then a Khalimsky continuous map $f:\left(X, T_{X}^{n}\right) \rightarrow\left(Y, T_{Y}^{n}\right)$ need not preserve digital connectivity [10]. This is one of the reasons why we go together a Khalimsky topological structure and digital connectivity (see Example 3.3 of the current paper). Thus the recent papers $[10,14,15]$ studied a Khalimsky topological space $\left(X, T_{X}^{n}\right)$ with one of the $k$-adjacency relations of $\mathbf{Z}^{n}$. Further, we have developed special kinds of continuities related to the preservation of digital connectivity as well as Khalimsky continuity such as KD- $\left(k_{0}, k_{1}\right)-,\left(k_{0}, k_{1}\right)$ - and $\mathrm{K}-\left(k_{0}, k_{1}\right)$-continuities [10], and KD- $\left(k_{0}, k_{1}\right)-,\left(k_{0}, k_{1}\right)$ - and $\mathrm{K}-\left(k_{0}, k_{1}\right)$-homeomorphisms [10].

By computer topology is now meant the mathematical recognition of a set in $\mathbf{Z}^{n}$ with some reasonable topological structure and $k$-adjacency relations of $\mathbf{Z}^{n}$, e.g. a development of tools implementing topological concepts for use in science and engineering.

In this paper, by using several computer topological continuities between Khalimsky $n_{0} \mathrm{D}$ - and $n_{1} \mathrm{D}$-subspaces, we can study various properties of several kinds of $k$-retracts in computer topology and further, study extension problems of these continuous maps in relation with several kinds of $k$-retracts.

This paper is organized as follows. In Section 2 we provide some basic notions. In Section 3 we establish several computer topological continuities and investigate their various properties. In Section 4 several kinds of $k$-retracts are introduced and studied with several sorts of computer topological homeomorphisms. In Section 5 we study extension problems of these several continuities in relation with these $k$-retracts. Finally, in Section 6 we conclude the paper with a summary.

\section{Preliminaries}

In digital topology a set $X \subset \mathbf{Z}^{n}$ with $k$-adjacency, briefly $(X, k)$, has been usually considered in a quadruple $\left(\mathbf{Z}^{n}, k, \bar{k}, X\right)$, where $n \in \mathbf{N}, k$ represents an adjacency relation for $X$ and $\bar{k}$ represents an adjacency relation for $\mathbf{Z}^{n}-$ $X[17,18,20]$. In this paper a (binary) set $X \subset \mathbf{Z}^{n}$ can be assumed in $\left(\mathbf{Z}^{n}, k, 2 n, X\right)$ or $\left(\mathbf{Z}^{n}, 2 n, 3^{n}-1, X\right)$ with $k \neq 2 n$ except for $(\mathbf{Z}, 2,2, X)$ because of the digital connectivity paradox related to digital Jordan curve theorem [18]. 
In order to study a multi-dimensional set $X \subset \mathbf{Z}^{n}$ with $k$-adjacency denoted by $(X, k)$, we need to recall the $k$-adjacency relations of $\mathbf{Z}^{n}$ as follows. Motivated by 4- and 8-adjacencies of a 2D digital space, and 6-, 18- and 26adjacencies of a 3D digital space $[18,20]$, the $k$-adjacency relations of $\mathbf{Z}^{n}$ are induced from the following operator [6] (see also [10]):

For a natural number $m$ with $1 \leq m \leq n$, two distinct points

$$
p=\left(p_{1}, p_{2}, \ldots, p_{n}\right) \text { and } q=\left(q_{1}, q_{2}, \ldots, q_{n}\right) \in \mathbf{Z}^{n}
$$

are $k(m, n)$-(briefly, $k$ - or $\left.k_{m^{-}}\right)$adjacent if

- there are at most $m$ indices $i$ such that $\left|p_{i}-q_{i}\right|=1$ and

- for all other indices $i$ such that $\left|p_{i}-q_{i}\right| \neq 1, p_{i}=q_{i}$.

In this operator $k:=k(m, n)$ is the number of points $q$ that are $k$-adjacent to a given point $p$ according to the numbers $m$ and $n$ in $\mathbf{N}$, where " $:=$ " means equal by definition. Indeed, this $k(m, n)$-adjacency is another presentation of the $k$-adjacency of [6] and a generalization of the $k$-adjacency of $2 \mathrm{D}$ and 3D digital images in [20]. Consequently, this operator leads to the $k$-adjacency relations of $\mathbf{Z}^{n}$ [8] (for more details, see [14]):

Proposition 2.1 ([12]). There are $k$-adjacency relations of $\mathbf{Z}^{n}$ :

$$
k:=k(m, n)=\sum_{i=n-m}^{n-1} 2^{n-i} C_{i}^{n}, \text { where } C_{i}^{n}=\frac{n !}{(n-i) ! i !}
$$

In this paper each set $X \subset \mathbf{Z}^{n}$ is considered to be a subspace $\left(X, T_{X}^{n}\right)$ induced from the Khalimsky $n \mathrm{D}$ space $\left(\mathbf{Z}^{n}, T^{n}\right)$. Indeed, motivated by the Alexandroff topological space [1], Khalimsky line topology on $\mathbf{Z}$ is induced from the family of the set $\{\{2 n+1\},\{2 m-1,2 m, 2 m+1\} \mid m, n \in \mathbf{Z}\}$, which is a basis of the Khalimsky line topology on $\mathbf{Z}$. For $\{a, b\} \subset \mathbf{Z}$ with $a \lesseqgtr b$, $[a, b]_{\mathbf{z}}=\{a \leq n \leq b \mid n \in \mathbf{Z}\}$ with 2-adjacency is considered to be a subspace of $(\mathbf{Z}, T)$ or a set related to the presentation of a sequence in Section 3. Further, the product topology on $\mathbf{Z}^{n}$ is derived from the Khalimsky line topology on $\mathbf{Z}, n \geq 2$. Then the topology on $\mathbf{Z}^{n}$ is called the product Khalimsky topology on $\mathbf{Z}^{n}$, and we use the notation $\left(\mathbf{Z}^{n}, T^{n}\right)$. A topological space is said to be a $T_{\frac{1}{2}}$-space if each singleton is either open or closed [4] and further, it turns out that $(\mathbf{Z}, T)$ is a $T_{\frac{1}{2}}$-space. If $n \geq 2$, then the Khalimsky $n \mathrm{D}$ space $\left(\mathbf{Z}^{n}, T^{n}\right)$ is a $T_{0}$-space instead of a $T_{\frac{1}{2}}$-space [17].

For the Khalimsky $n D$ space $\left(\mathbf{Z}^{n}, T^{n}\right)$ let us recall that a point $x=\left(x_{1}, x_{2}, \ldots\right.$, $\left.x_{n}\right) \in \mathbf{Z}^{n}$ is open if all coordinates are odd, and closed if each of the coordinates is even [17]. These points are called pure and the other points in $\mathbf{Z}^{n}$ is called mixed. In all subspaces of $\left(\mathbf{Z}^{n}, T^{n}\right), n \geq 2$, of Figures $1,2,3,4,5,6,7$ and 8, the symbols $\mathbf{\square}$ and $\bullet$ mean a pure closed point and a mixed point, respectively. Besides, a jumbo dot stands for a pure open point. 


\section{Several continuities in computer topology}

Let us begin with a brief review of various continuities and homeomorphisms for studying computer topological spaces of Definition 1 . We now recall the following basic terminology. We say that a set of lattice points is $k$-connected if it is not a union of two disjoint non-empty sets that are not $k$-adjacent to each other [18]. For a digital space $(X, k)$, two points $x, y \in X$ are called $k$ connected if there is a sequence $\left(x_{i}\right)_{i \in[0, m]_{\mathbf{z}}} \subset X$ such that $x_{0}=x, x_{m}=y$ and further, $x_{i}$ and $x_{i+1}$ are $k$-adjacent, $i \in[0, m-1]_{\mathbf{z}}, m \geq 1$ [20]. The number $m$ is called the length of this $k$-path [20]. For an adjacency relation $k$, a simple $k$-path in $X$ is a sequence $\left(x_{i}\right)_{i \in[0, m]_{\mathbf{Z}}} \subset X$ such that $x_{i}$ and $x_{j}$ are $k$-adjacent if and only if either $i=j \pm 1$ [18]. For $(X, k)$, a point $x \in X$ is called isolated if it is not $k$-connected with any point in $X$ [18]. A simple closed $k$-curve with $l$ elements in $\mathbf{Z}^{n}$ is said to be a sequence $\left(w_{i}\right)_{i \in[0, l-1]_{\mathbf{Z}}} \subset \mathbf{Z}^{n}$ such that $w_{i}$ and $w_{j}$ are $k$-adjacent if and only if either $j=i+1(\bmod l)$ or $i=j+1(\bmod l)$ [18] and is denoted by $S C_{k}^{n, l}[8]$.

For a set $X \subset \mathbf{Z}^{n}$, let us consider the subspace induced from the Khalimsky $n \mathrm{D}$ space $\left(\mathbf{Z}^{n}, T^{n}\right)$ denoted by $\left(X, T_{X}^{n}\right)$. Further, we recall the following presentation.

Definition 1 ([10]). A space $\left(X, T_{X}^{n}\right)$ with $k$-adjacency is called a computer topological space (briefly, a space if not confused) and is denoted by $X_{n, k}:=$ $\left(X, k, T_{X}^{n}\right)$.

Obviously, for a set $Y \subset \mathbf{Z}$, the subspace $\left(Y, T_{Y}\right) \subset(\mathbf{Z}, T)$ is always assumed to have 2-adjacency. For a space $X_{n, k}$ we need two kinds of $k$-neighborhoods for establishing several continuities in computer topology (see Definitions 2 and $3)$.

Definition 2 ([7] (see also [10])). Let $(X, k)$ be a digital space, $x, y \in X$, and $\varepsilon \in \mathbf{N}$.

(1) By $l_{k}(x, y)$ we denote the length of a shortest simple $k$-path from $x$ to $y$ in $X$. Further, we define $l_{k}(x, y)=\infty$ if there is no $k$-path from $x$ to $y$.

(2) By $N_{k}(x, \varepsilon)$ we denote the set $\left\{y \in X: l_{k}(x, y) \leq \varepsilon\right\} \cup\{x\}$. This $N_{k}(x, \varepsilon)$ is called a digital $k$-neighborhood of $x$ with radius $\varepsilon$ [7] (see also [8]).

According to Definition 2, for any $\varepsilon \in \mathbf{N}$ we observe that $N_{k}(x, \varepsilon)=\{x\}$ if the point $x$ is isolated.

Let us now consider another $k$-neighborhood from the view point of computer topology.

Definition 3 ([7] (see also $[10,15]))$. For $X_{n, k}$ we define the following notion.

(1) A subset $V$ of $X$ is called a topological neighborhood of $x$ if there exists $O_{x} \in T_{X}^{n}$ such that $x \in O_{x} \subseteq V$ as usual.

(2) If the set $N_{k}(x, \varepsilon)$ in Definition 2 is a (Khalimsky) topological neighborhood of $x$ in $\left(X, T_{X}^{n}\right)$, then this set is called a (Khalimsky topological) $k$-neighborhood of $x$ with radius $\varepsilon$ and is denoted by $N_{k}^{*}(x, \varepsilon)$. 
Remark 3.1. The current $k$-neighborhood $N_{k}^{*}(x, \varepsilon) \subset X_{n, k}$ is different from the digital $k$-neighborhood $N_{k}(x, \varepsilon)$ of Definition 2 and further, $N_{k}^{*}(x, \varepsilon)$ absolutely depends on the computer topological structure of $X_{n, k}$.

Example 3.2. Consider the space $A_{2, k}, k \in\{4,8\}$, where $A=\left\{x_{i} \mid i \in\right.$ $\left.[0,11]_{\mathbf{z}}\right\}$ in Figure 1 . Then some computer topological $k$-neighborhoods can be considered, as follows.

Since the smallest open set containing the point $x_{8}$ is the set $\left\{x_{8}, x_{9}\right\}$, we obtain the following:

$$
\begin{aligned}
& N_{8}^{*}\left(x_{8}, 1\right)=\left\{x_{7}, x_{8}, x_{9}, x_{10}\right\}, N_{8}^{*}\left(x_{8}, 2\right)=\left\{x_{6}, x_{7}, x_{8}, x_{9}, x_{10}, x_{11}\right\}, \text { and } \\
& N_{4}^{*}\left(x_{8}, 1\right)=\left\{x_{8}, x_{9}\right\} .
\end{aligned}
$$

Meanwhile, since the singleton $\left\{x_{0}\right\}$ is an open set and the point $x_{0}$ is not 4-connected with any point in $A$, we obtain $N_{4}^{*}\left(x_{0}, \varepsilon\right)=\left\{x_{0}\right\}, \varepsilon \in \mathbf{N}$.

But we obtain $N_{4}\left(x_{5}, 1\right)=\left\{x_{3}, x_{5}\right\}$ and $N_{4}\left(x_{5}, 2\right)=\left\{x_{2}, x_{3}, x_{4}, x_{5}\right\}$. Since the smallest open set containing the point $x_{5}$ is the set $\left\{x_{2}, x_{3}, x_{4}, x_{5}, x_{6}\right\}$, for any $\varepsilon \in \mathbf{N}$, no $N_{4}^{*}\left(x_{5}, \varepsilon\right)$ exists because there is no open set $O_{x_{5}} \in T_{X}^{2}$ such that $O_{x_{5}} \subset N_{4}\left(x_{5}, \varepsilon\right)$, where $O_{x_{5}}$ means an open set including the point $x_{5}$.

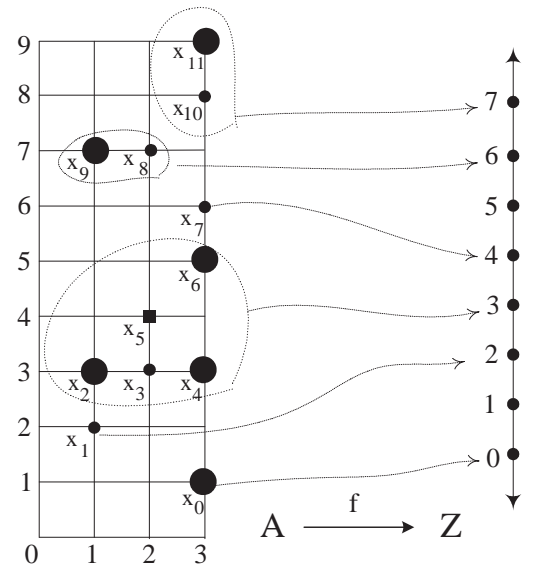

FIGURE 1. Comparison between Khalimsky continuity and digital-(8,2)-continuity.

In Khalimsky topology, we can observe that a Khalimsky continuous map $f$ : $X_{n_{0}, k_{0}} \rightarrow Y_{n_{1}, k_{1}}$ need not preserve the $k_{0}$-connectivity into the $k_{1}$-connectivity [10]. To be specific, as discussed in [10, 15], consider the space $A_{2,8}$ and the map $f: A_{2,8} \rightarrow(\mathbf{Z}, T)$ in Figure 1. While $f$ is Khalimsky continuous at the point $x_{8}$, it cannot preserve the 8 -connectedness of $A_{2,8}$ into the 2 -one of $(\mathbf{Z}, T)$. This is one of the reasons why we study a set $X \subset \mathbf{Z}^{n}$ with special kinds of continuities comprising both Khalimsky continuity and digital continuity. Indeed, in both 
digital topology and computer topology, for a map $f: X_{n_{0}, k_{0}} \rightarrow Y_{n_{1}, k_{1}}$ the preservation of the $k_{0}$-connectivity into the $k_{1}$-one by the map $f$ should be required to study digital spaces and computer topological spaces. Thus various properties of digital continuity have been studied $[3,11,16,20]$ and further, several kinds of computer topological $\left(k_{0}, k_{1}\right)$-continuities were introduced in [10]. Let us now recall several continuities in computer topology.

Definition 4 ([10]). A function $f: X_{n_{0}, k_{0}} \rightarrow Y_{n_{1}, k_{1}}$ is said to be Khalimsky continuous with digital $\left(k_{0}, k_{1}\right)$-continuity (briefly, KD- $\left(k_{0}, k_{1}\right)$-continuous) at a point $x \in X_{n_{0}, k_{0}}$ if

(1) $f$ is Khalimsky continuous at the point $x$ and

(2) $f\left(N_{k_{0}}(x, 1)\right) \subset N_{k_{1}}(f(x), 1)$.

Further, we say that a map $f: X_{n_{0}, k_{0}} \rightarrow Y_{n_{1}, k_{1}}$ is $\mathrm{KD}-\left(k_{0}, k_{1}\right)$-continuous if it is $\mathrm{KD}-\left(k_{0}, k_{1}\right)$-continuous at every point $x \in X_{n_{0}, k_{0}}$.

Motivated by digital continuity of [3, 20], Definition 4(2) is established and called digital $\left(k_{0}, k_{1}\right)$-continuity in [6] (see also $\left.[8,11]\right)$. It can be used without any limitation of studying a multi-dimensional digital space in $\mathbf{Z}^{n}, n \in \mathbf{N}$. Further, this digital $\left(k_{0}, k_{1}\right)$-continuity can be essentially used for studying the preservation of the local $k_{0}$-property into the local $k_{1}$-property [13] as well as the almost pasting property [16]. In addition, we can observe that none of the conditions (1) and (2) of Definition 4 implies the other (see Example 3.3) if $n_{0}, n_{1} \in \mathbf{N}-\{1\}$.

Example 3.3. Consider $A_{2,8}$ in Figure 1, where $A=\left\{x_{i}\right\}_{i \in[0,11]_{\mathbf{z}}} \subset \mathbf{Z}^{2}$ in Figure 1. Let us consider the map $f: A \rightarrow \mathbf{Z}$ (see Figure 1) for which $f\left(x_{0}\right)=$ $0, f\left(x_{1}\right)=2, f\left(x_{7}\right)=4, f\left(x_{8}\right)=f\left(x_{9}\right)=6, f\left(A_{1}\right)=\{3\}$ and $f\left(A_{2}\right)=\{7\}$, where $A_{1}=\left\{x_{2}, x_{3}, x_{4}, x_{5}, x_{6}\right\}$ and $A_{2}=\left\{x_{10}, x_{11}\right\}$.

While the map $f$ is Khalimsky continuous, it cannot be digitally $(8,2)$ continuous at the point $x_{8} \in A$ contrary to Definition 4(2) because we obtain $f\left(N_{8}\left(x_{8}, 1\right)\right) \nsubseteq N_{2}(6,1)$.

Although KD- $\left(k_{0}, k_{1}\right)$-continuity is so helpful to study $X_{n, k}$, it need not be sufficient for the study of a space $X_{n, k}$ in computer topology. Thus, by using a computer topological $k$-neighborhood, we need to establish another continuity that is different from KD- $\left(k_{0}, k_{1}\right)$-continuity as follows.

Definition 5 ([10] (see also [15])). A function $f: X_{n_{0}, k_{0}} \rightarrow Y_{n_{1}, k_{1}}$ is said to be $\left(k_{0}, k_{1}\right)$-continuous at a point $x \in X$ if for any $N_{k_{1}}^{*}(f(x), \varepsilon) \subset Y$ there is $\delta \in \mathbf{N}$ and $N_{k_{0}}^{*}(x, \delta) \subset X$ such that

$$
f\left(N_{k_{0}}^{*}(x, \delta)\right) \subset N_{k_{1}}^{*}(f(x), \varepsilon),
$$

where for some $\varepsilon \in \mathbf{N}, N_{k_{1}}^{*}(f(x), \varepsilon)$ is assumed to be existed. Further, we say that a map $f: X_{n_{0}, k_{0}} \rightarrow Y_{n_{1}, k_{1}}$ is $\left(k_{0}, k_{1}\right)$-continuous if it is $\left(k_{0}, k_{1}\right)$-continuous at every point $x \in X_{n_{0}, k_{0}}$.

In Definition 5 if such a neighborhood $N_{k_{1}}^{*}(f(x), \varepsilon)$ does not exist in $Y_{n_{1}, k_{1}}$, then we obviously say that the map $f$ cannot be $\left(k_{0}, k_{1}\right)$-continuous at the 
point $x \in X_{n_{0}, k_{0}}$. The $\left(k_{0}, k_{1}\right)$-continuity of Definition 5 is equivalent to the following presentation [10] (see also [15]):

$$
f\left(N_{k_{0}}^{*}(x, r)\right) \subset N_{k_{1}}^{*}(f(x), s),
$$

where the number $r$ is the least element of $\mathbf{N}$ such that $N_{k_{0}}^{*}(x, r)$ contains an open set including the point $x$ (so $N_{k_{0}}^{*}(x, r)=N_{k_{0}}(x, r)$ ) and $s$ is the least element of $\mathbf{N}$ such that $N_{k_{1}}^{*}(f(x), s)$ contains an open set including the point $f(x)\left(\right.$ so $\left.N_{k_{1}}^{*}(f(x), s)=N_{k_{1}}(f(x), s)\right)$.

Indeed, the current $\left(k_{0}, k_{1}\right)$-continuity of Definition 5 is different from the digital $\left(k_{0}, k_{1}\right)$-continuity of Definition $4(2)$ and can be used for studying a computer topological space $X_{n, k}$. Although both $\mathrm{KD}-\left(k_{0}, k_{1}\right)$ - and $\left(k_{0}, k_{1}\right)$ continuities can contribute to the study of computer topological space, they need not be sufficient for studying $X_{n, k}$, either. Thus, the following notion can be used in studying spaces $X_{n, k}$.

Definition 6 ([10]). A function $f: X_{n_{0}, k_{0}} \rightarrow Y_{n_{1}, k_{1}}$ is said to be Khalimsky $\left(k_{0}, k_{1}\right)$-continuous (briefly, $\mathrm{K}$ - $\left(k_{0}, k_{1}\right)$-continuous) at a point $x \in X_{n_{0}, k_{0}}$ if

(1) $f:\left(X, T_{X}^{n_{0}}\right) \rightarrow\left(Y, T_{Y}^{n_{1}}\right)$ is Khalimsky continuous at the point $x$, and

(2) the map $f$ satisfies Definition 5 at the point $x$.

Further, we say that a map $f: X_{n_{0}, k_{0}} \rightarrow Y_{n_{1}, k_{1}}$ is $\mathrm{K}$ - $\left(k_{0}, k_{1}\right)$-continuous if the map $f$ is $\mathrm{K}$ - $\left(k_{0}, k_{1}\right)$-continuous at every point $x \in X_{n_{0}, k_{0}}$.

We observe that none of the conditions (1) and (2) of Definition 6 implies the other if $n_{0}, n_{1} \in \mathbf{N}-\{1\}$ (see Remark 3.4). In addition, since Definition 4(2) and Definition 6(2) are so different from each other, we clearly observe that $\mathrm{KD}-\left(k_{0}, k_{1}\right)$-continuity and $\mathrm{KD}$ - $\left(k_{0}, k_{1}\right)$-continuity cannot be equivalent to each other.

Remark 3.4. (1) We now show that Definition 6(2) does not imply Definition 6(1). Precisely, consider $X:=\left(a_{i}\right)_{i \in[0,5]_{\mathbf{z}}}$ with 26-adjacency and the map $f: X \rightarrow \mathbf{Z}$ in Figure 2(a) for which $f(A)=\{3\}, f\left(a_{3}\right)=2, f\left(a_{5}\right)=4$, where $A=\left\{a_{0}, a_{1}, a_{2}, a_{4}\right\}$. While the map $f$ satisfies Definition $6(2)$, it cannot be Khalimsky continuous at the point $a_{4}$. To be specific, let us now examine K$(26,2)$-continuity of the map $f$ at the point $a_{4}$ and let us remind that $N_{26}^{*}\left(a_{4}, 1\right)$ is the total set $X$. While for $N_{2}^{*}(3,1)$ we obtain $f\left(N_{26}^{*}\left(a_{4}, 1\right)\right) \subset N_{2}^{*}(3,1)$, which implies Definition $6(2)$ at the point $a_{4}, f$ cannot be Khalimsky continuous at the point $a_{4}$ because Khalimsky continuity of $f$ at the point $a_{4}$ should require $f\left(N_{26}^{*}\left(a_{4}, 1\right)\right) \subset\{3\} \in(\mathbf{Z}, T)$.

(2) We now show that Definition 6(1) need not imply Definition 6(2):

(Case 1) Let us consider $Y_{n_{1}, k_{1}}$ of Definition 6 to be $(\mathbf{Z}, T)$.

Consider the map $f$ in Example 3.3. Then the map $f: A_{2,8} \rightarrow(\mathbf{Z}, T)$ in Figure 1 cannot be $\mathrm{K}-(8,2)$-continuous at the point $x_{8} \in A$ contrary to Definition $6(2)$. More precisely, for $N_{8}^{*}\left(x_{8}, 1\right)=\left\{x_{7}, x_{8}, x_{9}, x_{10}\right\}$ we can observe $f\left(N_{8}^{*}\left(x_{8}, 1\right)\right) \nsubseteq$ $N_{2}^{*}(6,1)$.

(Case 2) Let us consider $Y_{2,8}$ or $Y_{2,4}$ according to Definition 6 . Assume that $A=\left\{x_{i}\right\}_{i \in[1,11]_{\mathbf{z}}} \subset \mathbf{Z}^{2}$ and $B=\left\{y_{j}\right\}_{j \in[1,6]_{\mathbf{z}}} \subset \mathbf{Z}^{2}$ (see Figure 
2(b)). Next, consider the map $f: A \rightarrow B$ for which $f\left(x_{1}\right)=y_{1}, f\left(x_{2}\right)=$ $f\left(x_{3}\right)=f\left(x_{4}\right)=y_{2}, f\left(x_{5}\right)=f\left(x_{6}\right)=y_{3}, f\left(x_{7}\right)=y_{4}, f\left(x_{8}\right)=y_{5}$, and $f\left(x_{9}\right)=$ $f\left(x_{10}\right)=f\left(x_{11}\right)=y_{6}$ (see Figure $\left.2(\mathrm{~b})\right)$.

Assume $A_{2,8}$ or $A_{2,4}$, and $B_{2,8}$ or $B_{2,4}$. Then, while the map $f$ is Khalimsky continuous, $f$ cannot be K-(8,8)-, K-(4,4)-, $(8,4)-,(4,8)$-continuous. To be specific, although $x_{1}$ and $x_{2}$ are 4-adjacent, the images $f\left(x_{1}\right)$ and $f\left(x_{2}\right)$, which is not 4 -adjacent, are obviously 8 -adjacent. While $x_{7}$ and $x_{8}$ are 8 -adjacent, both their images of $f$ are not 8 -adjacent. Thus $f$ cannot be K- $(8,8)$-continuous at the point $x_{7}$.

Similarly, we can observe that the map $f$ cannot be K-(4,4)-, $(8,4)-,(4,8)-$ continuous, either.

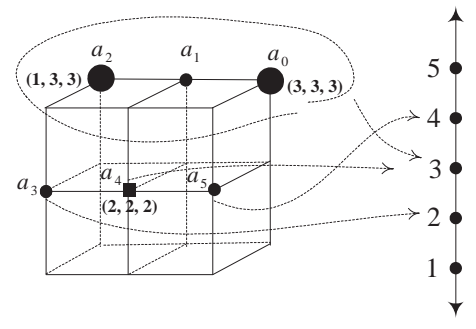

(a) $\mathrm{X} \longrightarrow \mathrm{Z}$

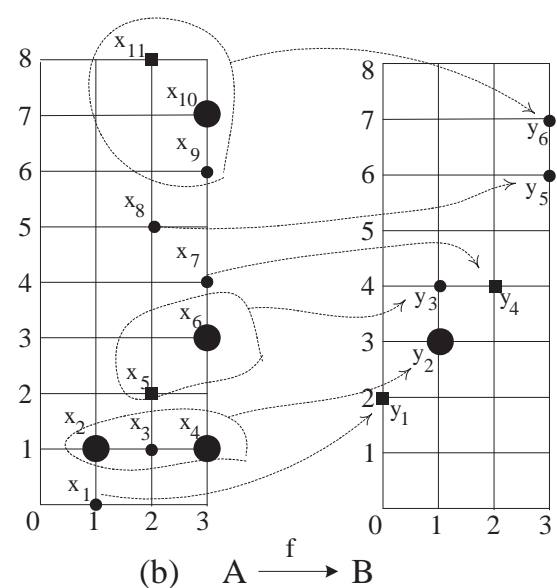

(b) $\mathrm{A} \stackrel{\mathrm{f}}{\longrightarrow} \mathrm{B}$

Figure 2. Non-existence of $\mathrm{K}$ - $\left(k_{0}, k_{1}\right)$-continuity, $k_{0}, k_{1} \in\{4,8\}$.

As referred above, for a map $f: X_{n_{0}, k_{0}} \rightarrow Y_{n_{1}, k_{1}}$ if either of $N_{k_{0}}^{*}(x, 1) \subset$ $X_{n_{0}, k_{0}}$ and $N_{k_{1}}^{*}(f(x), 1) \subset Y_{n_{1}, k_{1}}$ does not exist, then we may have some difficulty in defining both $\mathrm{K}-\left(k_{0}, k_{1}\right)$-continuity and $\left(k_{0}, k_{1}\right)$-continuity of the map $f$ at the point $x$. Thus we observe that $\mathrm{K}-\left(k_{0}, k_{1}\right)$-continuity is different from $\mathrm{KD}-\left(k_{0}, k_{1}\right)$-continuity (see Remark 3.5$)$.

Remark 3.5. If a space $X_{n, k}$ is not $k$-connected, then for some point $x \in X$ and any $\varepsilon \in \mathbf{N}$ we may not have $N_{k}^{*}(x, \varepsilon)$. For instance, consider $A_{2,4}$ instead of $A_{2,8}$ in Figure 2(b). Then for any $\varepsilon \in \mathbf{N}$, no $N_{4}^{*}\left(x_{5}, \varepsilon\right)$ exists. While $f: A_{2,4} \rightarrow B_{2,8}$ is Khalimsky continuous, it cannot satisfy Definition $6(2)$ at the point $x_{5}$, which means that the map $f$ cannot be a K- $(4,8)$-continuous map. Meanwhile, we observe that $f$ is $\mathrm{KD}$ - $(4,8)$-continuous at the point $x_{5}$.

With some hypothesis, we can compare the above-mentioned computer topological continuities, as follows. 
Theorem 3.6. For $\left(X, T_{X}^{n}\right)$ and the Khalimsky line $(\mathbf{Z}, T)$ if a map $f: X \rightarrow \mathbf{Z}$ is Khalimsky continuous at a pure closed point $x \in X$, then $f$ is also KD$\left(3^{n}-1,2\right)-,\left(3^{n}-1,2\right)-$, and $K-\left(3^{n}-1,2\right)$-continuous at the point.

Proof. If a point $x$ is a pure closed point, then the smallest open neighborhood of $x$ is $N_{3^{n}-1}^{*}(x, 1)$. Thus for any point $x_{1} \in N_{3^{n}-1}^{*}(x, 1)$, we obtain $f\left(x_{1}\right) \in$ $N_{2}^{*}(f(x), 1)$ so that $f\left(N_{3^{n}-1}^{*}(x, 1)\right) \subset N_{2}^{*}(f(x), 1)$ by the Khalimsky continuity of $f$ (see the point $x_{5}$ in Figure 1$)$. Consequently, the map $f$ is also K- $\left(3^{n}-1,2\right)$ continuous at the point.

Similarly, the proofs of $\left(3^{n}-1,2\right)-$, and K- $\left(3^{n}-1,2\right)$-continuities of the map $f$ can be done by using the same method used to prove the above KD$\left(3^{n}-1,2\right)$-continuity of $f$.

Due to the above-mentioned several continuities in computer topology, we obtain the following several categories in computer and digital topologies [10].

(1) Let us consider the Khalimsky topological category, denoted by KTC, consisting of two things:

- A class of objects $\left(X, T^{n}\right)$;

- KTC has Khalimsky continuous maps as morphisms.

(2) Let us consider the KD-topological category, denoted by KDTC, consisting of two things:

- A class of objects $X_{n, k}$;

- KDTC has KD- $\left(k_{0}, k_{1}\right)$-continuous maps as morphisms.

(3) Let us consider the computer topological category, denoted by CTC, consisting of two things:

- A class of objects $X_{n, k}$;

- CTC has $\left(k_{0}, k_{1}\right)$-continuous maps as morphisms.

(4) Let us consider the Khalimsky computer topological category, denoted by KCTC, consisting of two things:

- A class of objects $X_{n, k}$;

- KCTC has K- $\left(k_{0}, k_{1}\right)$-continuous maps as morphisms.

(5) Finally, let us consider the digital-topological category, denoted by DTC, consisting of two things:

- A class of objects $(X, k)$ in $\mathbf{Z}^{n}$;

- DTC has digitally $\left(k_{0}, k_{1}\right)$-continuous maps as morphisms.

In Sections 4 and 5, by using these computer or digital topological categories, we will establish several kinds of $k$-retracts and study extension problems of the just above continuities from (1) to (5) in relation with several $k$-retracts in Section 4.

\section{Various $k$-retracts and their properties in relation with computer topological homeomorphisms}

For a subset $X \subset \mathbf{Z}^{n}$ or $\mathbf{Z}$, by Theorem 3.6, an extension problem of a $\mathrm{K}$ $\left(3^{n}-1,2\right)$-continuous map $f: X_{n, 3^{n}-1} \rightarrow(\mathbf{Z}, T)$ at a pure point can be easily 
studied. We now investigate extension problems of the other cases $f: X_{n, k} \rightarrow$ $Y_{n_{1}, k_{1}}$.

Motivated by the notion of retract in [2], the paper [3] develops the notion of digital $k$-retract for the study of an extension problem of digital continuity:

Definition $7([3])$. In DTC, we say that a digitally $k$-continuous map $r$ : $\left(X^{\prime}, k\right) \rightarrow(X, k)$ is a digital $k$-retraction if

(1) $X \subset X^{\prime}$, and

(2) $r(x)=x$ for all $x \in X$.

Then we say that $X$ is a digital $k$-retract of $X^{\prime}$.

In Definition 7 , a point $x \in X^{\prime}-X$ is said to be digitally $k$-retractable.

Similarly, in KTC, the Khalimsky topological version of the retraction of Definition 7 can be established, as follows.

In KTC, we say that a Khalimsky continuous map $\left.r:\left(X^{\prime}, T_{X^{\prime}}^{n}\right) \rightarrow X, T_{X}^{n}\right)$ is a Khalimsky retraction if

(1) $\left(X, T_{X}^{n}\right)$ is a Khalimsky topological subspace of $\left(X^{\prime}, T_{X^{\prime}}^{n}\right)$, and

(2) $r(x)=x$ for all $x \in X$.

Then we call $X$ a Khalimsky retract of $X^{\prime}$.

Motivated by the digital $k$-retract of Definition 7 and the above-mentioned Khalimsky retraction, we now introduce several kinds of $k$-retracts for studying spaces $X_{n, k}$ (see Definitions 8, 10 and 12) and study their properties in relation with several homeomorphisms in Definitions 9, 11 and 13, as follows.

Definition 8 ([14]). In KDTC, we say that a KD- $k$-continuous map $r: X_{n, k}^{\prime} \rightarrow$ $X_{n, k}$ is a KD-k-retraction if

(1) $X_{n, k} \subset X_{n, k}^{\prime}$, and

(2) $r(x)=x$ for all $x \in X_{n, k}$.

Then we say that $X_{n, k}$ is a KD-k-retract of $X_{n, k}^{\prime}$. Further, we say that a point $x \in X_{n, k}^{\prime}-X_{n, k}$ is KD-k-retractable.

Example 4.1. Consider a space $X_{2,4}$ in Figure $3\left(\right.$ a), where $X=\left\{x_{i}\right\}_{i \in[1,11]_{\mathbf{z}}}$. Assume that $X^{\prime}=X \cup\left\{a_{1}, a_{2}\right\}$ in Figure 3(a). Let us now consider a space $Y_{2,4}$ in Figure 3(b), where $Y=\left\{y_{i}\right\}_{i \in[1,13]_{\mathrm{z}}}$ and assume $Y^{\prime}=Y \cup\left\{b_{1}, b_{2}, b_{3}\right\}$ in Figure 3(b). Then $X_{2,8}$ is a KD-8-retract of $X_{2,8}^{\prime}$ and $Y_{2,4}$ is a KD-4-retract of $Y_{2,4}^{\prime}$ in such a way that: $a_{1} \rightarrow x_{8}, a_{2} \rightarrow x_{11}$, and the other points in $X_{2,8}$ are remained.

Further, $b_{1} \rightarrow y_{4}, b_{2} \rightarrow y_{6}, b_{3} \rightarrow y_{12}$, and the other points in $Y$ are also remained.

Definition 9 ([10]). In KDTC, a function $f: X_{n_{0}, k_{0}} \rightarrow Y_{n_{1}, k_{1}}$ is said to be a $\mathrm{KD}$ - $\left(k_{0}, k_{1}\right)$-homeomorphism if

(1) the map $f$ is bijective, and

(2) the map $f$ is $\mathrm{KD}$ - $\left(k_{0}, k_{1}\right)$-continuous and further, $f^{-1}$ is $\mathrm{KD}$ - $\left(k_{1}, k_{0}\right)$ continuous. Then we say that $X_{n_{0}, k_{0}}$ is $\operatorname{KD}-\left(k_{0}, k_{1}\right)$-homeomorphic to $Y_{n_{1}, k_{1}}$. 
(a)

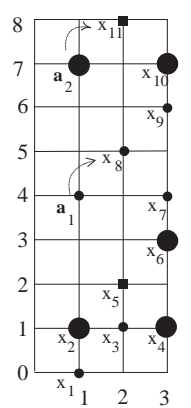

(b)

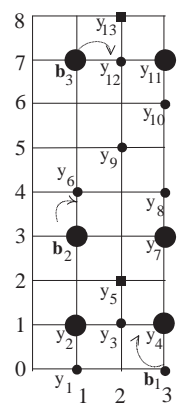

FiguRE 3. (a) KD-8-retract (b) KD-4-retract.

Example 4.2. Consider the simple closed 8-curves with eight elements $S C_{8}^{2,8}:=$ $\left(w_{i}\right)_{i \in[0,7]_{\mathbf{z}}}, T:=\left(e_{i}\right)_{i \in[0,7]_{\mathbf{z}}}$ (see Figure 4). The map $g: S C_{8}^{2,8} \rightarrow T_{2,8}$ given by $g\left(w_{i}\right)=e_{i}$ is a $\mathrm{KD}$ - $(8,8)$-homeomorphism because the singletons $\left\{e_{2}\right\}$ and $\left\{e_{6}\right\}$ are also open sets in $T_{2,8}$.

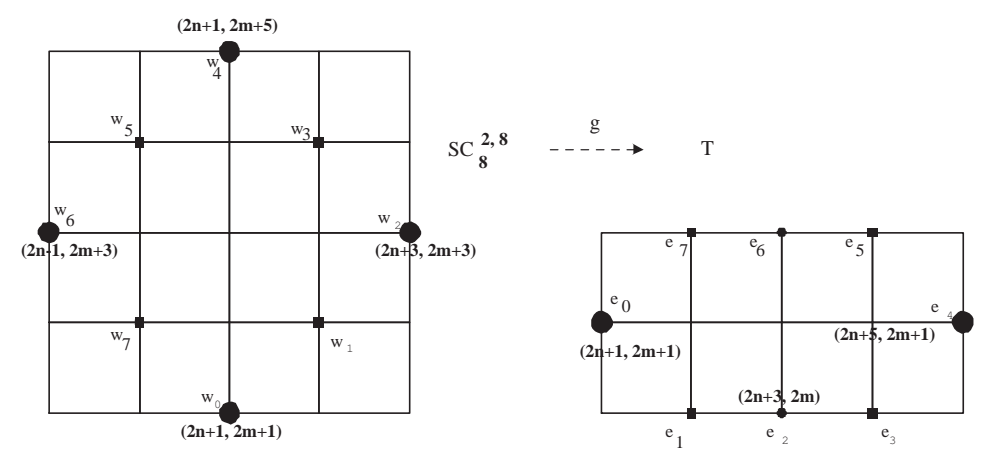

FiguRE 4. KD-(8,8)-homeomorphism.

Motivated by the digital isomorphic property of the digital $k$-retract in [3], we obtain the following property of a KD- $\left(k_{0}, k_{1}\right)$-homeomorphism.

Theorem 4.3. Let $X_{n, k}$ be a KD-k-retract of $X_{n, k}^{\prime}$ and let $h: X_{n, k}^{\prime} \rightarrow Y_{n_{1}, k_{1}}$

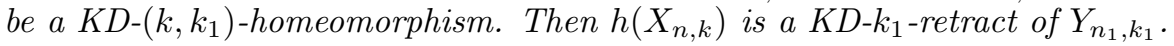

Proof. Let $r: X_{n, k}^{\prime} \rightarrow X_{n, k}$ be a KD-k-retraction. Then $h \circ r \circ h^{-1}: Y_{n_{1}, k_{1}} \rightarrow$ $h\left(X_{n, k}\right)$ is a KD- $k_{1}$-retraction because the composition of $\mathrm{KD}-\left(k_{1}, k\right)-$, KD$(k, k)$ - and $\mathrm{KD}-\left(k, k_{1}\right)$-continuous maps is also $\mathrm{KD}-\left(k_{1}, k_{1}\right)$-continuous.

By using the similar method as Definition 8, in CTC we obtain the following: 
Definition 10. In CTC, we say that a $k$-continuous map $r: X_{n, k}^{\prime} \rightarrow X_{n, k}$ is a $k$-retraction if

(1) $X_{n, k} \subset X_{n, k}^{\prime}$, and

(2) $r(x)=x$ for all $x \in X_{n, k}$.

Then we say that $X_{n, k}$ is a $k$-retract of $X_{n, k}^{\prime}$. Further, we say that a point $x \in X_{n, k}^{\prime}-X_{n, k}$ is $k$-retractable.

Definition 11 ([10]). In CTC, a function $f: X_{n_{0}, k_{0}} \rightarrow Y_{n_{1}, k_{1}}$ is said to be a $\left(k_{0}, k_{1}\right)$-homeomorphism if

(1) the map $f$ is bijective, and

(2) the map $f$ is a $\left(k_{0}, k_{1}\right)$-continuous map and further, $f^{-1}$ is a $\left(k_{1}, k_{0}\right)$ continuous map. Then we say that $X_{n_{0}, k_{0}}$ is $\left(k_{0}, k_{1}\right)$-homeomorphic to $Y_{n_{1}, k_{1}}$.

By using the same method used to prove Theorem 4.3, we obtain the following:

Theorem 4.4. In CTC, let $X_{n, k}$ be a k-retract of $X_{n, k}^{\prime}$ and let $h: X_{n, k}^{\prime} \rightarrow$ $Y_{n_{1}, k_{1}}$ be a $\left(k, k_{1}\right)$-homeomorphism. Then $h\left(X_{n, k}\right)$ is a $k_{1}$-retract of $Y_{n_{1}, k_{1}}$.

In KCTC, by using the same method as Definitions 8 and 10, we can also establish the notion of $\mathrm{K}-k$-retraction:

Definition 12. In KCTC, we say that a $\mathrm{K}$ - $(k, k)$-continuous map $r: X_{n, k}^{\prime} \rightarrow$ $X_{n, k}$ is a $\mathrm{K}-k$-retraction if

(1) $X_{n, k} \subset X_{n, k}^{\prime}$, and

(2) $r(a)=a$ for all $a \in X_{n, k}$.

Then we say that $X_{n, k}$ is a K-k-retract of $X_{n, k}^{\prime}$. Further, we say that the point $a \in X_{n, k}^{\prime}-X_{n, k}$ is $\mathrm{K}$ - $k$-retractable.

Example 4.5. Consider the two spaces in Figure 3. Then $X_{2,8}$ is a K-8-retract of $X_{n, k}^{\prime}$ and $Y_{2,4}$ is a $\mathrm{K}$-4-retract of $Y_{2,4}^{\prime}$ in the same way as Example 4.1.

Definition $13([10])$. In KCTC, a function $f: X_{n_{0}, k_{0}} \rightarrow Y_{n_{1}, k_{1}}$ is said to be a $\mathrm{K}$ - $\left(k_{0}, k_{1}\right)$-homeomorphism if

(1) the map $f$ is bijective, and

(2) the map $f$ is a $\mathrm{K}$ - $\left(k_{0}, k_{1}\right)$-continuous map and further, $f^{-1}$ is a $\mathrm{K}$ - $\left(k_{1}, k_{0}\right)$ continuous map. Then we say that the space $X_{n_{0}, k_{0}}$ is $\mathrm{K}-\left(k_{0}, k_{1}\right)$-homeomorphic to $Y_{n_{1}, k_{1}}$.

By using the same method used to prove the above KD- $(8,4)$-homeomorphism of $f$ in Example 4.2, we can observe that $S C_{8}^{2,8}$ is $\mathrm{K}-(8,8)$ - and $(8,8)$ homeomorphic to $T_{2,8}$. By using the same method used to prove Theorem 4.3, we obtain the following:

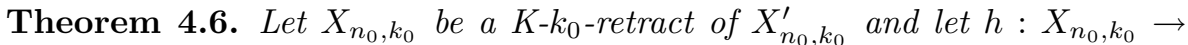
$Y_{n_{1}, k_{1}}$ be a $K-\left(k_{0}, k_{1}\right)$-homeomorphism. Then $h\left(X_{n_{0}, k_{0}}\right)$ is a K- $k_{1}$-retract of $Y_{n_{1}, k_{1}}$. 
Example 4.7. Put $X=X^{\prime}-\left\{x_{1}, x_{5}, x_{9}\right\}$ (see Figure 5). Then we observe that $X_{2,8}$ is a K-8-retract of $X_{2,8}^{\prime}$. Consider a K-(8,8)-homeomorphism $h: X_{2,8} \rightarrow$ $Y_{2,8}$ defined by $f\left(x_{i}\right)=y_{i}, i \in[1,9]_{\mathbf{z}}$. Then $h(X)_{2,8}$ is obviously a K-8-retract of $Y_{2,8}$, where $h(X)=Y-\left\{y_{1}, y_{5}, y_{9}\right\}$.
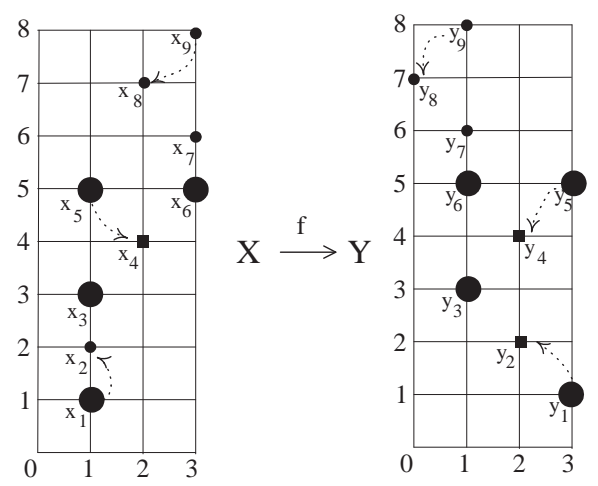

FiguRE 5. Computer topological homeomorphism in relation with K-8-retract.

In addition, the map $f$ in Figure 5 also guarantees Theorems 4.3 and 4.6. We now say that properties that when possessed by a space in $\mathbf{Z}^{n}$ are also possessed by KD- $\left(k_{0}, k_{1}\right)-,\left(k_{0}, k_{1}\right)$-, and $\mathrm{K}-\left(k_{0}, k_{1}\right)$-homeomorphisms are called $K D$ - $\left(k_{0}, k_{1}\right)$-, $\left(k_{0}, k_{1}\right)$ - and KD-( $\left.k_{0}, k_{1}\right)$-homeomorphic properties, respectively. Obviously, $k$-connectedness is KD- $k$-homeomorphic property.

Theorem 4.8. Each of $K D-k$-, $k$ - and $K$ - $k$-retracts holds the reflexivity and the transitivity.

\section{Extension problems of several continuities in computer topology}

In classical topology [2], we recall the following: Let $\left(X^{\prime}, T^{\prime}\right)$ be a topological space and $(X, T)$ a subspace of $\left(X^{\prime}, T^{\prime}\right)$. Then $(X, T)$ is a retract of $\left(X^{\prime}, T^{\prime}\right)$ if and only if every continuous map $f:(X, T) \rightarrow\left(Y, T_{1}\right)$ has a continuous map $F: X^{\prime} \rightarrow Y$ such that $\left.F\right|_{X}=f$ for any $\left(Y, T_{1}\right)$ [2]. Consequently, in KTC instead of KDTC, CTC and KCTC, every Khalimsky continuous map $f:\left(X, T_{X}^{2}\right) \rightarrow\left(Y, T_{Y}^{n_{1}}\right)$ has an extension $F:\left(X^{\prime}, T_{X^{\prime}}^{2}\right) \rightarrow\left(Y, T_{Y}^{n_{1}}\right)$ such that $\left.F\right|_{X}=f$ for any $\left(Y, T_{Y}^{n_{1}}\right)$ if and only if $X$ is a Khalimsky retraction of $X^{\prime}$.

Unlike the extension property, in view of Remarks 3.4 and 3.5, the extension problems of the several computer topological continuities in Section 3 have their intrinsic features (see Theorems 5.1 and 5.3, and Corollary 5.4). Thus we need to study extension problems of the computer topological continuities in relation with the computer topological $k$-retracts in Section 4 . We now establish a criterion of a subspace $X_{n, k}$ that is equivalent to the condition that for each of 
$\mathrm{KD}-\left(k, k_{1}\right)$-, $\left(k, k_{1}\right)$-, and $\mathrm{K}$ - $\left(k, k_{1}\right)$-continuous maps $f: X_{n, k} \rightarrow Y_{n_{1}, k_{1}}$, there is a space $X_{n, k}^{\prime}$ containing the space $X_{n, k}$ and its corresponding $\mathrm{KD}-\left(k, k_{1}\right)$-, $\left(k, k_{1}\right)$-, and $\mathrm{K}$ - $\left(k, k_{1}\right)$-continuous map $F: X_{n, k}^{\prime} \rightarrow Y_{n_{1}, k_{1}}$ such that $\left.F\right|_{X_{n, k}}=f$ for any $Y_{n_{1}, k_{1}}$.

Theorem 5.1. In KDTC, $X_{n, k}$ is a KD-k-retract of $X_{n, k}^{\prime}$ if and only if every $K D$ - $\left(k, k_{1}\right)$-continuous map $f: X_{n, k} \rightarrow Y_{n_{1}, k_{1}}$ has a KD-( $\left.k, k_{1}\right)$-continuous $\operatorname{map} F: X_{n, k}^{\prime} \rightarrow Y_{n_{1}, k_{1}}$ such that $\left.F\right|_{X_{n, k}}=$ f for any $Y_{n_{1}, k_{1}}$.

Proof. Let $r: X_{n, k}^{\prime} \rightarrow X_{n, k}$ be a KD- $k$-retraction and $f: X_{n, k} \rightarrow Y_{n_{1}, k_{1}}$ a KD$\left(k, k_{1}\right)$-continuous function. Then the composition $F:=f \circ r: X_{n, k}^{\prime} \rightarrow Y_{n_{1}, k_{1}}$ is a $\mathrm{KD}-\left(k, k_{1}\right)$-continuous map that is an extension of $f$.

Conversely, suppose that every KD- $\left(k, k_{1}\right)$-continuous map $f: X_{n, k} \rightarrow Y_{n_{1} k_{1}}$ has a KD- $\left(k, k_{1}\right)$-continuous extension $F: X_{n, k}^{\prime} \rightarrow Y_{n_{1} k_{1}}$ for every $Y_{n_{1} k_{1}}$. Then the identity map $1_{X_{n, k}}$ has a KD- $\left(k, k_{0}\right)$-continuous extension $r: X_{n, k}^{\prime} \rightarrow X_{n, k}$. Thus $X_{n, k}$ is a KD-k-retract of $X_{n, k}^{\prime}$.

To guarantee Theorem 5.1, let us consider the space $X_{2,8}$ in Figure 6. In addition, consider another space $Y_{2, k}, k \in\{4,8\}$ in Figure 6 . Since $X_{2,8}$ cannot be $k$-retract of $Y_{2, k}, k \in\{4,8\}$, we observe that there is no KD-(8,8)-continuous extension of $1_{X_{2,8}}$ into the map $f: Y_{2, k} \rightarrow X_{2,8}$ in Figure 6 .

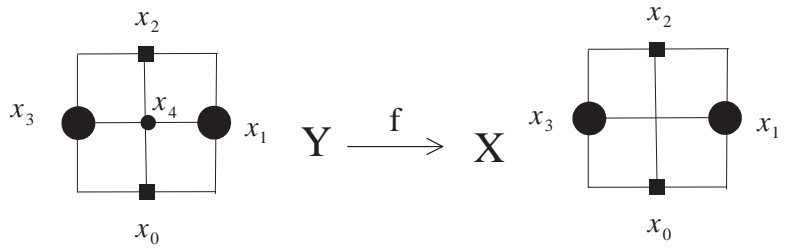

Figure 6. Non-existence of an extension of KD-(8,8)-continuity.

Example 5.2. In Figure 7(a), for two sets $X=\left\{x_{i}\right\}_{i \in[0,8]_{\mathbf{z}}}$ and $Y=\left\{y_{i}\right\}_{i \in[0,3]_{\mathbf{z}}}$, consider a map $g: X_{2,4} \rightarrow Y_{2,8}$ for which $g\left(\left\{x_{0}, x_{1}, x_{2}, x_{3}, x_{6}\right\}\right)=\left\{y_{0}\right\}, g\left(x_{4}\right)=$ $y_{2}, g\left(x_{5}\right)=y_{1}$, and $g\left(\left\{x_{7}, x_{8}\right\}\right)=\left\{y_{3}\right\}$. Then $g$ is a KD-(4,8)-continuous map.

Assume $X^{\prime}=X \cup\left\{c_{1}\right\}$ and consider $X_{2,4}^{\prime}$. Then $X_{2,4}$ is a KD-4-retract of $X_{2,4}^{\prime}$ in such a way that $c_{1} \rightarrow x_{4}$ and the other points of $X_{2,4}^{\prime}$ are remained, where $c_{1}=(1,0)$. Then there is a map $G: X_{2,4}^{\prime} \rightarrow Y_{2,8}$ such that $\left.G\right|_{X_{2,4}}$ and $G\left(\left\{c_{1}\right\}\right)=\left\{y_{2}\right\}$, i.e., $G$ is a KD-(4,4)-continuous extension of $g$.

Meanwhile, consider the space in Figure $7(\mathrm{~b})$. Assume $D_{2,4}$ and the map $f: X_{2,4} \rightarrow \mathbf{Z}$ for which $f\left(X_{1}\right)=\{3\}$ and $f\left(\left\{x_{7}, x_{8}\right\}\right)=\{-1\}$, where $D:=$ $X \cup\left\{d_{1}\right\}$ and $X=\left\{x_{i}\right\}_{i \in[0,8]_{\mathbf{z}}}$ and $d_{1}=(2,4)$ in Figure $7(\mathrm{~b})$. Then, in KDTC, there is no extension of $f$ on $D_{2,4}$ because $X_{2,4}$ cannot be a KD-4-retract of $D_{2,4}$. To be specific, in Figure 7(b), consider the set $D:=X \cup\left\{d_{1}\right\}$ and assume 
$D_{2,4}$. Then $X_{2,4}$ cannot be a 4 -retract of $D_{2,4}$ because the smallest open set containing the point $d_{1}$ is the set $\left\{x_{2}, x_{3}, x_{6}, d_{1}, x_{7}, x_{8}\right\}$. Thus, in KDTC there is no extended map $F: D_{2,4} \rightarrow \mathbf{Z}$ such that $\left.F\right|_{X_{2,4}}=f$ (see Figure $7($ b)) owing to the non-existence of a KD-4-retraction from $D_{2,4}$ onto $X_{2,4}$ at the point $d_{1}$.

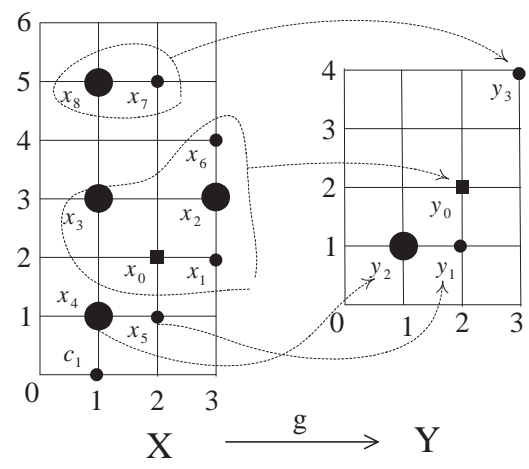

(a)

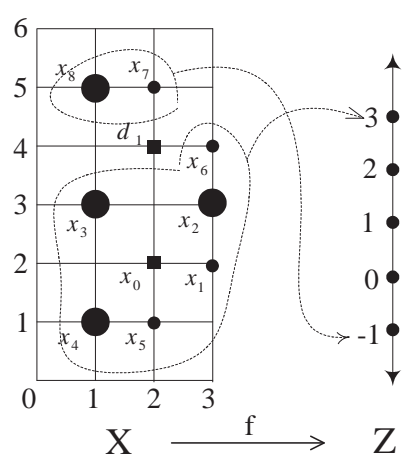

(b)

FiguRE 7. (a) Existence of KD-(8,8)- and K-(8,8)-extension maps; (b) Non-existence of KD- $(8,8)$ - and K-(8,8)-extension maps .

By using the same method used to prove Theorem 5.1, in CTC we obtain the following:

Theorem 5.3. In CTC, let $X_{n, k}^{\prime}$ and $Y_{n_{1}, k_{1}}$ be $k$ - and $k_{1}$-connected spaces, respectively. Let $X_{n, k}$ be a k-connected subspace of $X_{n, k}^{\prime}$. Then $X_{n, k}$ is a $k$ retract of $X_{n, k}^{\prime}$ if and only if every $\left(k, k_{1}\right)$-continuous map $f: X_{n, k} \rightarrow Y_{n_{1}, k_{1}}$ has a $\left(k, k_{1}\right)$-continuous map $F: X_{n, k}^{\prime} \rightarrow Y_{n_{1}, k_{1}}$ such that $\left.F\right|_{X_{n, k}}=f$ for any $Y_{n_{1}, k_{1}}$.

Before we prove Theorem 5.3, we need to discuss the assumption of Theorem 5.3 on the $k$-connectedness of $X_{n, k}$ and the $k_{1}$-connectedness of $Y_{n_{1}, k_{1}}$. If the hypothesis is omitted, we may not have some computer topological $k$ - or $k_{1}$ neighborhood of some points in $X_{n, k}^{\prime}$ and $Y_{n_{1}, k_{1}}$ (see Remark 3.5). Hereafter, we always assume that each space $X_{n, k}$ in CTC and $\mathrm{KCTC}$ is $k$-connected.

Proof of Theorem 5.3. The proof can be done by the same method as that of Theorem 5.1 by replacing both a KD- $\left(k, k_{1}\right)$-continuous function and a KD- $k$ retraction in KDTC into both a $\left(k, k_{1}\right)$-continuous function and a $k$-retraction in CTC, respectively.

By combining Theorems 5.1 and 5.3, in KCTC we obtain the following: 
Corollary 5.4. In $K C T C$, let $A_{n_{0}, k_{0}}^{\prime}$ and $B_{n_{1}, k_{1}}$ be $k_{0}$ - and $k_{1}$-connected, respectively. For a $k_{0}$-connected space $A_{n_{0}, k_{0}} \subset A_{n_{0}, k_{0}}^{\prime}$, a $K$-( $\left(k_{0}, k_{1}\right)$-continuous map $f: A_{n_{0}, k_{0}} \rightarrow B_{n_{1}, k_{1}}$ has an extended map $F: A_{n_{0}, k_{0}}^{\prime} \rightarrow B_{n_{1}, k_{1}}$ for any $B_{n_{1}, k_{1}}$ that is also $K$ - $\left(k_{0}, k_{1}\right)$-continuous if and only if $A_{n_{0}, k_{0}}$ is a $K$ - $k_{0}$-retract of $A_{n_{0}, k_{0}}^{\prime}$.

Let us consider the domain of the map $f$ in Corollary 5.4 to be a subspace in $\left(\mathbf{Z}^{2}, T^{2}\right)$, as follows.

Example 5.5. In Figure 5, assume $A=X-\left\{x_{1}, x_{5}, x_{9}\right\}, B=Y-\left\{y_{1}, y_{5}, y_{9}\right\}$. Consider $f: A_{2,8} \rightarrow B_{2,8}$ given by $f\left(x_{i}\right)=y_{i}, i \in\{2,3,4,6,7,8\}$. Then $f$ is a $\mathrm{K}-(8,8)$-continuous map. Further, the space $A_{2,8}$ is a K-8-retract of $X_{2,8}$. Thus, there is an extension map $F: X_{2,8} \rightarrow B_{2,8}$ defined by $F\left(x_{1}\right)=y_{2}, F\left(x_{5}\right)=y_{4}$, $F\left(x_{9}\right)=y_{8}$, and $\left.F\right|_{X_{2,8}}=f$, which is a K- $(8,8)$-continuous extension of $f$.

Let us consider the domain of the map $f$ in Corollary 5.4 as a subspace in $\left(\mathbf{Z}^{3}, T^{3}\right)$, as follows.

Example 5.6. Assume the space $X=A \cup\{(2,2,2)\}$ (see Figure 8), where $A=\{(1,2,2),(1,3,3),(2,3,3),(3,3,3),(3,2,2)\}$. In addition, consider the map $f: A_{3,18} \rightarrow(\mathbf{Z}, T)$ for which $f((1,2,2))=2, f((1,3,3))=3, f((2,3,3))=$ $3, f((3,3,3))=3$ and $f((3,2,2))=4$ so that $f$ is a $\mathrm{K}-(18,2)$-continuous map. Assume $X=A \cup\{(2,2,2)\}$. Then there is no extended K-(18,2)-continuous map $F: X_{3,18} \rightarrow(\mathbf{Z}, T)$ because the point $(2,2,2) \in X$ cannot be K-18retractable.

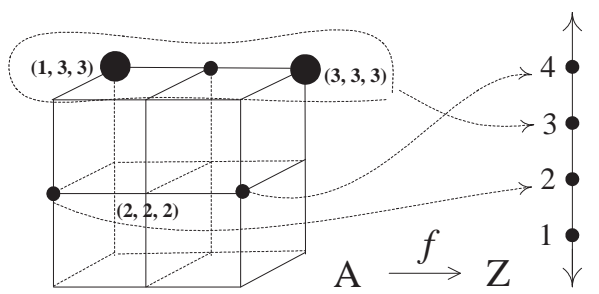

Figure 8. Non-existence of KD-(18,2)-, (18,2)-, and K$(18,2)$-continuities.

In KDTC and CTC, let us now consider the map $f$ in Figure 8. Obviously, it is also a KD- $(18,2)$ - as well as an $(18,2)$ - continuous map. By using the same method as Example 5.6, by Theorems 5.1 and 5.3, we can observe that the map $f$ cannot be extended to the above $F$ that is KD- $(18,2)$ - or $(18,2)$ - continuous because the point $(2,2,2) \in X$ is neither KD-18- nor 18-retractable. 


\section{Summary}

All results in this paper can be presented from the view point of digital topology in terms of the forgetful functor from KDTC, CTC, and KCTC into DTC: Assume forgetful functors from KDTC, CTC, and KCTC into DTC, denoted by $F^{*}$ : KDTC, CTC, and KCTC $\rightarrow$ DTC. To be specific, for a space $X_{n, k}$ in KDTC, CTC, and KCTC, $F^{*}$ is defined in such a way that $F^{*}\left(X_{n, k}\right)=$ $(X, k) \in$ DTC. Besides, for a KD- $\left(k_{0}, k_{1}\right)$-, $\left(k_{0}, k_{1}\right)$-, or a $\mathrm{K}-\left(k_{0}, k_{1}\right)$-continuous map $f$, we obtain $F^{*}(f)=f \in$ DTC. This can be essentially applied to digital geometry and computer science. The results of Sections 4 and 5 are computer topological versions in KDTC, CTC, and KCTC of digital $k$-retract properties in DTC [3].

\section{References}

[1] P. Alexandorff, Diskrete Räume, Mat. Sb. 2 (1937), 501-518.

[2] K. Borsuk, Theory of Retracts, Polish Scientific publisher, Warsaw, 1967.

[3] L. Boxer, Digitally continuous functions, Pattern Recognition Letters 15 (1994), 833839.

[4] W. Dunham, $T_{1 / 2}$-spaces, Kyungpook Math. J. 17 (1977), no. 2, 161-169.

[5] G. Gierz, K. H. Hofmann, K. Keimel, J. D. Lawson, M. Mislove, and D. S. Scott, A Compendium of Continuous Lattices, Springer, Berlin-Heidelberg-New York, 1980.

[6] S. E. Han, Computer topology and its applications, Honam Math. J. 25 (2003), no. 1, $153-162$.

[7] Comparison between digital continuity and computer continuity, Honam Math. J. 26 (2004), no. 3, 331-339.

[8] - Non-product property of the digital fundamental group, Inform. Sci. 171 (2005), no. 1-3, 73-91.

[9] Strong $k$-deformation retract and its applications, J. Korean Math. Soc. 44 (2007), no. 6, 1479-1503.

[10] Continuities and homeomorphisms in computer topology and their applications, J. Korean Math. Soc. 45 (2008), no. 4, 923-952.

[11] Equivalent $\left(k_{0}, k_{1}\right)$-covering and generalized digital lifting, Inform. Sci. 178 (2008), no. 2, 550-561.

[12] ing Vision 31 (2008), no. 1, 1-16.

[13] _ Map preserving local properties of a digital image, Acta Appl. Math. 104 (2008), no. $2,177-190$.

[14] $K D$ - $\left(k_{0}, k_{1}\right)$-homotopy equivalence and its applications, J. Korean Math. Soc. 47 (2010), no. 5, 1031-1054.

[15] S. E. Han and N. D. Georgiou, On computer topological function space, J. Korean Math. Soc. 46 (2009), no. 4, 841-857.

[16] I.-S. Kim, S. E. Han, and C. J. Yoo, The pasting property of digital continuity, Acta Appl. Math. 110 (2010), no. 1, 399-408.

[17] E. Khalimsky, R. Kopperman, and P. R. Meyer, Computer graphics and connected topologies on finite ordered sets, Topology Appl. 36 (1990), no. 1, 1-17.

[18] T. Y. Kong and A. Rosenfeld, Topological Algorithms for the Digital Image Processing, Elsevier Science, Amsterdam, 1996.

[19] E. Melin, Extension of continuous functions in digital spaces with the Khalimsky topology, Topology Appl. 153 (2005), no. 1, 52-65. 
[20] A. Rosenfeld, Arcs and curves in digital pictures, J. Assoc. Comput. Mach. 20 (1973), $81-87$.

Faculty of Liberal Education

Institute of Pure and Applied Mathematics

Chonbuk National University

JeOnJu 561-756, KoreA

E-mail address: sehan@jbnu.ac.kr 